\title{
Grundversorgung: Der Weg aus der Krise führt auch über die MPA*
}

Die meisten Hausärzte und Hausärztinnen in der Schweiz sind dauerhaft überlastet. Um zusätzliche Kapazitäten zu schaffen, sind ihre Leistungen auf mehr Schultern zu verteilen und die Betreuung im (Praxis-)Team zu verstärken. Besonders dafür geeignet ist die Begleitung von chronisch kranken Patienten durch die Medizinische Praxisassistentin. Das Problem: Der Arzt kann diese Leistungen nicht abrechnen. Dies ist so rasch wie möglich zu ändern, damit die MPA endlich den Wert erhält, den sie längst verdient.

Thomas Rosemann ${ }^{a}$,

Emil Schalch ${ }^{b}$,

Beatrice Birnbaumc,

Urs Zanonid

a Prof. Dr. med., Direktor des Instituts für Hausarztmedizin und Versorgungsforschung, Universität Zürich

b Dr. med., Präsident der kantonalen MPA-Delegierten der FMH, Präsident der OdA Berufsbildung MPA

c MPA, Ausbilderin FA, Vorstand Schweizerischer Verband Medizinischer PraxisAssistentinnen SVA

d MPH, Geschäftsführer mediX zürich und Projektleiter Futuro Betreuungsprogramm

\footnotetext{
* Siehe zum Thema dieses Artikels auch das Editorial dieser Ausgabe von Zentralvorstandsmitglied Ernst Gähler.
}

Korrespondenz: Dr. med. Emil Schalch Facharzt für Allgemeinmedizin FMH Fischmattstrasse 2 CH-6315 Oberägeri emil.schalch@hin.ch
Am 10. April 2010 war ein Artikel im Wissen-Teil des Tages-Anzeigers wie folgt betitelt: «Bei Problemen und Nebenwirkungen: Fragen Sie Ihre Arztgehilfin!» Die Kernaussage des Beitrags: Durch die Aufwertung der Medizinischen Praxisassistentin (MPA) kann die Grundversorgung - besonders die Betreuung von chronisch Kranken - qualitativ verbessert, effizienter und letztlich kostengünstiger werden.

Dass die Hausarztmedizin neu organisiert werden muss, weil die bestehenden Kapazitäten dauerhaft überlastet sind, ist inzwischen unbestritten. Und die Perspektiven bleiben düster: Das Schweizerische Gesundheitsobservatorium geht davon aus, dass sich die Lücke zwischen nachgefragten und angebotenen Konsultationen beim Hausarzt jedes Jahr um eine halbe Million vergrössert. Als Folge davon findet eine Abwanderung in teure Spitalambulatorien statt.

\section{Ohne MPA geht es nicht}

Ein prioritärer Ansatz zur Neuorganisation der hausärztlichen Tätigkeit besteht darin, die Leistungen auf mehr Schultern zu verteilen und die Betreuung im (Praxis-)Team zu verstärken. Besonders geeignet dafür ist die Begleitung von chronisch kranken Patienten, da sie sehr zeitintensiv ist (Beratung, Schulung, Motivation). Anderseits umfasst sie viele Routineverrichtungen wie Blutdruck messen, Füsse begutachten oder Vibrationsempfinden prüfen, die sich an nichtärztliches Personal - namentlich die MPA - delegieren lassen.

Es ist unbestritten, dass die Betreuung von chronisch Kranken zu den Domänen der Hausarztmedizin gehört; je nach Praxis entfallen 60 bis 80 Prozent der Konsultationen auf diesen Bereich. Viele Betroffene haben überdies mehr als eine chronische Erkrankung, wie eine aktuelle Auswertung des Instituts für Hausarztmedizin und Versorgungsforschung der Universität Zürich zeigt: Die Prävalenz der Multimorbidität bei hausärztlichen Patienten ist mit 16\% (von rund

\section{Zusammenfassung}

Die Zeichen für die Hausarztmedizin stehen momentan schlecht. Und die Perspektiven sind ebenso düster: Das Schweizerische Gesundheitsobservatorium geht davon aus, dass sich die Lücke zwischen nachgefragten und angebotenen Konsultationen beim Hausarzt jedes Jahr um eine halbe Million vergrössert. Als Folge davon wandern Patienten in teure Spitalambulatorien ab.

Das muss nicht sein: Um zusätzliche Kapazitäten zu schaffen, sind die Leistungen von Hausärztinnen und Hausärzten auf mehr Schultern zu verteilen und die Betreuung im (Praxis-)Team zu verstärken. Besonders dafür geeignet ist die Begleitung von chronisch kranken Patienten. Hier kann die Medizinische Praxisassistentin (MPA) zahlreiche verantwortungsvolle Aufgaben übernehmen: Beratung, Schulung, Coaching, Monitoring. Sie kennt die Praxisabläufe, spricht die Sprache der Patienten und hat häufig ein ausgeprägtes Vertrauensverhältnis zu ihnen. Eine spezifische Weiterbildung in Chronic Care Management lässt sich leicht in die bestehenden Strukturen integrieren. Das Problem: Der Arzt kann die Betreuungsleistungen der MPA bei chronisch Kranken nicht abrechnen. Dies ist so rasch wie möglich zu ändern, damit die MPA endlich als effiziente Ressource zur Stärkung der Grundversorgung genutzt werden kann. 
125000 Behandlungen) deutlich höher als die Prävalenz der häufigsten Einzeldiagnose (Hypertonie mit $12 \%)$.

Aus medizinischer Sicht spricht folglich vieles dafür, einen substantiellen Teil des Chronic Care Management an nichtärztliches Personal in der Praxis zu delegieren - so weit sind sich die Fachleute einig. Hingegen gehen die Meinungen auseinander, welche nichtärztlichen Fachpersonen ins ambulante Chronic Care Management integriert werden sollen. Manchenorts werden Leute aus der Pflege bevorzugt. So schreibt der Bundesrat in seinem Bericht «Zur aktuellen Situation der ärztlichen Grundversorgung»: «In diesem Zusammenhang sollte die Tertiarisierung der Pflegeberufe so genutzt werden, dass Pflegefachleute zur Entlastung und Ergänzung der ärztlichen Grundversorger beitragen können.»

Diese Haltung übergeht, dass mit den MPAs bereits personelle Ressourcen in der Hausarztpraxis vorhanden sind, die verantwortungsvolle Aufgaben übernehmen. Und die sich - ergänzt um eine spezifische Weiterbildung - verstärkt fürs Chronic Care Management einsetzen lassen:

- Die MPA kennt die Praxisabläufe und die Arbeitsweise des Hausarztes oder der Hausärztin.

- Da sie die Sprache der Patienten spricht, kann sie auch komplexe Sachverhalte verständlich erklären. Zudem trauen sich die Betroffenen eher, der MPA vermeintlich «dumme» Fragen zu stellen.

- Oft hat die MPA ein ausgeprägtes Vertrauensverhältnis zu den Patienten: Sie kennt die Lebensgeschichte, die täglichen Sorgen und Nöte und häufig auch die Angehörigen. Viele ältere Patienten und multimorbide chronisch Kranke sind meist älter - sehen den Arzt immer noch als Autorität und haben Mühe, sich ihm zu öffnen.

\section{Never change a winning team}

Es ist deshalb nicht nachvollziehbar, weshalb der Bericht des Bundesrates die MPA mit keinem Wort erwähnt und allein auf Pflegefachpersonen setzt obwohl es heute schon zu wenig von ihnen gibt und sie primär für den Einsatz in stationären Einrichtungen ausgebildet werden. Folglich fehlt ihnen die Polyvalenz, die in der Arztpraxis unabdingbar ist, was die Arbeitsteilung und Teambildung deutlich erschwert.

Hausarzt und MPA hingegen sind bereits eingespielt; eine neue Rollenteilung im Chronic Care Management wird dadurch erleichtert. Es gibt inzwischen zahlreiche Belege, dass der Teamansatz mit verstärktem Einbezug der MPA sinnvoll und wirksam ist:

- Ein strukturiertes Monitoring durch Praxisassistentinnen bei Arthrose-Kranken führte zu einer verbesserten Lebensqualität und reduzierte die inadäquate Beanspruchung von Spezialisten und apparativer Medizin.

- Eine in den Annals of Internal Medicine erschienene Arbeit konnte zeigen, dass ein MPA-basierter
Teamansatz auch bei Patienten mit einer Major Depression eine signifikante Verbesserung der Erkrankung bewirkt.

- Eine Auswertung von Disease Management Programmen, in denen MPAs Diabetiker beraten und begleiten, ergab eine signifikant reduzierte Mortalität.

Zudem zeigt die überwiegende Mehrheit der Studien, dass die Patienten den vermehrten Einbezug von Praxisassistentinnen in der Arztpraxis schätzen und akzeptieren. Immerhin: Auch in der Schweiz gewinnt diese Option langsam an Bedeutung (siehe Kasten auf der folgenden Seite).

\section{Fundierte Weiterbildung als Voraussetzung}

Dabei ist es unbestritten, dass die MPA für die strukturierte Betreuung von multimorbiden, chronisch kranken Patienten zusätzliche Qualifikationen erwerben muss (Beratung, Schulung, Coaching, Monitoring). Nachdem die MPA-Ausbildung mit der jüngsten Revision der Bildungsverordnung auf eine zukunftsfähige Basis gestellt wurde, ziehen die gleichen Leute jetzt bei der Weiterbildung nach: 2008 gründeten sie die Organisation der Arbeitswelt OdA Bildung MPA mit dem Ziel, 2011 die ersten Berufsprüfungen zur Medizinischen Praxisleiterin mit Eidgenössischem Fachausweis durchzuführen.

Es liegt auf der Hand, die Weiterbildung in Chronic Care Management in diese Struktur zu integrieren und ebenfalls zu einem Eidgenössischen Fachausweis zu führen. Damit wird die MPA zwei Abschlüsse auf Tertiärstufe zur Wahl haben. Beide decken zentrale Anforderungen an die Hausarztmedizin der Zukunft ab:

- Die Medizinische Praxisleiterin kann Führungsaufgaben in grösseren Praxen wahrnehmen.

- Die Vertiefung in Chronic Care Management schafft die dringend benötigten Kapazitäten zur qualitativ besseren Betreuung von multimorbiden Patienten. Davon profitieren namentlich kleinere Landpraxen, die nach wie vor die Mehrheit der Hausarztpraxen in der Schweiz ausmachen.

\section{Gute Arbeit verdient fairen Lohn}

Damit der Arzt als Arbeitgeber und Praxisbetreiber aber bereit ist, in eine solche Weiterbildung zu investieren, muss er die Betreuungsleistungen der MPA bei chronisch Kranken abrechnen können. Dies ist heute nicht möglich, weshalb dieses Potential weitgehend ungenutzt bleibt.

Dabei sind chronische Krankheiten und die Multimorbidität riesige Belastungen fürs Versorgungssystem: Sie verursachen rund 70 Prozent der Gesundheitskosten in Europa und Amerika. Nach Schätzungen der WHO werden 2020 mindestens 60 Prozent aller Erkrankungen chronischer Natur sein; 43 Prozent der Gesamtbevölkerung und 70 Prozent der über 65-Jährigen werden an einer, häufig sogar an zwei und mehr chronischen Erkrankungen leiden. 
Ökonomisch betrachtet gibt es eine vergleichsweise einfache Antwort auf diese Herausforderung, wie OECD-Daten belegen: Je stärker die Hausarztorientierung in einem System ist, desto kostengünstiger ist es (die Daten deuten überdies auf eine höhere Lebensqualität der Patienten hin). Nur lässt sich die Zahl der Hausärzte und Hausärztinnen in der Schweiz höchstens über viele Jahre hinweg steigern. Deutlich rascher hingegen können zusätzliche Kapazitäten über die Aufwertung der MPA und eine neue Arbeitsteilung im Betreuungsteam geschaffen werden.

\section{Die FMH will es wissen}

Damit der Arzt die Leistungen der MPA zulasten der Grundversicherung abrechnen kann, stehen zwei Wege offen:

- eine neue Pflichtleistung «Chronic Care Management» (mit anschliessender Tarifierung);

- eigenständige TARMED-Positionen für Betreuungsleistungen bei chronisch Kranken durch nichtärztliches Personal (delegierte Leistungen).

Der FMH-Tarifdienst ist daran, einen entsprechenden Antrag an die Paritätische Tarifkommission auszuarbeiten. Die Möglichkeit, solche Leistungen ordentlich abrechnen zu dürfen, ist sowohl für die Patienten wie die Versicherer vorteilhaft:

- Chronisch Kranke können in ihrer vertrauten Umgebung von vertrauten Personen begleitet werden

\begin{abstract}
Aufwertung der MPA in der Schweiz
Diabetes-Seminare für MPAs: Der Schweizerische Verband Medizinischer PraxisAssistentinnen SVA bietet seit 2001 das Seminar «Betreuung von Langzeitpatienten -- Modul Diabetes» an (seit 2009 besteht ein vergleichbares Seminar für Rheuma). Die praxisbezogene Weiterbildung schliesst mit einer schriftlichen und praktischen Lernerfolgskontrolle ab. Jedes Jahr wird ein ERFA-Tag mit Fachinput und Neuigkeiten aus dem Bereich Diabetes angeboten. Inzwischen haben rund 140 MPAs dieses Seminar absolviert.

Die CARAT-Studie untersucht, ob der Einbezug der MPA in die Betreuung von Diabetikern die Einstellung von HbA1c, LDL und Blutdruck verbessert. Verglichen werden in dieser randomisierten Interventionsstudie Hausarztpraxen mit und ohne speziell geschulte MPA. Diese verfolgt mit einem strukturierten Bogen und einem einfachen Ampelschema die Behandlung des Patienten. Dem Hausarzt liegen somit alle nötigen Informationen für seine Entscheidungen strukturiert und übersichtlich vor, was ihm eine raschere Orientierung ermöglicht. Ähnliche Instrumente haben sich bei anderen Erkrankungen als effektiv erwiesen.
\end{abstract}

Das Futuro Betreuungsprogramm: Initiiert durch die Ärztenetze mediX bern und zürich, wird derzeit ein Hausarzt-basiertes Betreuungsprogramm für multimorbide, chronisch kranke Patienten unter Einbezug der speziell weitergebildeten MPA entwickelt. Das Programm wird allen Praxen und Versicherten zugänglich sein, unabhängig von der Zugehörigkeit zu einem Netz und vom Versicherungsmodell. Das Betreuungsprogramm kombiniert mehrere bewährte Konzepte des Chronic Care Management: Medical Home, Betreuung im Team, Selbstmanagement der Patienten. Unterstützt werden die Anwender durch intelligente IT-Instrumente, die mit den führenden Praxisinformationssystemen kompatibel sind. Die Weiterbildung der MPA (Beratung, Schulung, Coaching, Monitoring) wird mit einem Eidg. Fachausweis in Chronic Care Management abschliessen. Das Programm, das im Frühjahr 2012 starten soll, wird vom Institut für Hausarztmedizin der Universität Zürich implementiert und evaluiert.
(Medical-Home-Ansatz). Fast alle heutigen Betreuungsprogramme finden dagegen ausserhalb der Praxis statt; entsprechend tief sind die Patientenzahlen. Eigenständige TARMED-Positionen für delegierte Leistungen an die MPA werden dazu führen, dass deutlich mehr Betroffene adäquat betreut werden. Was unerlässlich ist, denn wissenschaftliche Untersuchungen zeigen, dass chronisch Kranke häufig nicht die Versorgung erhalten, die sie benötigen.

- Die Versicherer profitieren von deutlich mehr Transparenz. Die ärztlichen und nichtärztlichen Leistungen werden separiert und zu unterschiedlichen Tarifen abgerechnet. Zudem sprechen alle Daten dafür, dass ein strukturiertes, proaktives Chronic Care Management kosteneffektiv ist: Eine Literatur-Review des Instituts für Hausarztmedizin und Versorgungsforschung der Universität Zürich kommt zum Schluss: «Eine Kostenreduktion oder lower use of health care services liess sich in 26 von 37 untersuchten Studien (= 70\%) bei Diabetes, Herzinsuffizienz, Asthma und COPD nachweisen.»

\section{Eine lohnende Investition}

Taxpunkte für die Betreuung von chronisch Kranken durch nichtärztliches Personal anzustreben, ist also weder blauäugig noch vermessen. Selbstverständlich wird es 12 bis 18 Monate dauern, bis erste kostendämpfende Effekte messbar werden. Anderseits ist ein beachtlicher Return on Investment (ROI) zu erwarten: Die amerikanische Gesundheitsorganisation Kaiser Permanente schätzt, dass allein SelfmanagementProgramme einen ROI von 5:1 bis 10:1 aufweisen,

- bedingt durch eine Reduktion von Spital-, Notfallund Arztkonsultationen,

- ein besseres Gesundheitsverhalten der Teilnehmenden,

- eine bessere Selbstbehandlung,

- verbesserte objektive Gesundheitsparameter sowie - ein verbessertes subjektives Gesundheitsempfinden.

Um zu verhindern, dass die beantragten TARMED-Positionen als «unkontrollierte Mengenausausweitung» bestritten werden, sind sie an Auflagen zu knüpfen. Zum einen an den angestrebten Eidgenössischen Fachausweis in Chronic Care Management; anderseits kann die Nutzung auf besonders häufige Diagnosen beschränkt werden (Diabetes, Adipositas, Bluthochdruck, COPD, Asthma, Herzinsuffizienz, Koronare Herzkrankheit). Bei sehr komplexen Situationen ist das Betreuungsteam ohnehin mit weiteren Fachpersonen - ärztlichen wie nichtärztlichen - zu ergänzen.

Wer ernsthaft die Grundversorgung stärken will, wird alles unternehmen, um die MPA aufzuwerten. Seit Jahrzehnten ist sie gesundheitspolitisch praktisch inexistent und tariflich zur Infrastruktur degradiert. Höchste Zeit also, diese Ressource zu aktivieren und ihr - buchstäblich - den Wert zu geben, den sie verdient. 